\title{
Improved LIDT values for dielectric dispersive compensating mirrors applying ternary composites
}

\author{
T. Willemsen*a, ${ }^{\text {a,b }}$ S. Schlichting $;$ M. Gyamfi; M. Jupé $;$ H. Ehlers ${ }^{\mathrm{b}} ;$ U. Morgner ${ }^{\mathrm{a}}$ and D. Ristau ${ }^{\mathrm{a}, \mathrm{b}}$ \\ ${ }^{a}$ Institut für Quantenoptik, Leibniz University, QUEST, Welfengarten 1, 30167 Hanover, Germany; \\ ${ }^{\mathrm{b}}$ Laser Zentrum Hannover e.V., Hollerithallee 8, 30419 Hanover, Germany
}

\begin{abstract}
The present contribution is addressed to an improved method to fabricate dielectric dispersive compensating mirrors (CMs) with an increased laser induced damage threshold (LIDT) by the use of ternary composite layers. Taking advantage of a novel in-situ phase monitor system, it is possible to control the sensitive deposition process more precisely. The study is initiated by a design synthesis, to achieve optimum reflection and GDD values for a conventional high low stack $(\mathrm{HL})^{\mathrm{n}}$. Afterwards the field intensity is analyzed, and layers affected by highest electric field intensities are exchanged by ternary composites of $\mathrm{Ta}_{\mathrm{x}} \mathrm{Si}_{\mathrm{y}} \mathrm{O}_{\mathrm{z}}$. Both designs have similar target specifications whereby one design is using ternary composites and the other one is distinguished by a $(\mathrm{HL})^{\mathrm{n}}$. The first layers of the stack are switched applying in-situ optical broad band monitoring in conjunction with a forward re-optimization algorithm, which also manipulates the layers remaining for deposition at each switching event. To accomplish the demanded GDD-spectra, the last layers are controlled by a novel in-situ white light interferometer operating in the infrared spectral range. Finally the CMs are measured in a 10.000 on 1 procedure according to ISO 21254 applying pulses with a duration of $130 \mathrm{fs}$ at a central wavelength of $775 \mathrm{~nm}$ to determine the laser induced damage threshold.
\end{abstract}

Keywords: chirp mirror, IBS process, in situ GDD measurement, GDD, design synthesis, ultra fast optics, ternary composites, LIDT

\section{INTRODUCTION}

Industrial applications applying ultra short laser pulses (USP) in the femtosecond (fs) regime are of rapidly growing importance. Additionally, the next generation of Multi Petawatt laser systems will necessarily use USP-laser sources. For both, the commercial laser as well as fundamental research laser systems, the precise control of the laser beam parameters is crucial for the success and further development of those high end applications. As a major aspect the significant temporal pulse broadening suffered due to the natural dispersion of light in the USP regime has to be managed by optical components in form of pairs of prisms, gratings or dielectric dispersion compensating mirrors $(\mathrm{CMs})$ [1]. In contrast to prisms and gratings, these dielectric mirrors can be adjusted more compliantly to the specific laser requirements. A variety of CMs from the visible (VIS) to the near infra-red spectral range (NIR) are commercially available. Typically, CMs require total film thicknesses of the stacks of several micrometers $(\mu \mathrm{m})$ [2]. Usually, compared to conventional dielectric QWOT mirrors, the complex design structure of CMs leads to higher electrical field intensities inside the coating stack. Both, the increase in layer thickness and the higher internal field strength cause a reduction of the laser induced damage threshold (LIDT). An improved control of the field intensity may lead to higher LIDT values of the layer structure. One possibility is claimed by the reduction of the index of refraction of layers with highest field intensity. Generally, the internal field strength in conjunction with the damage threshold of the corresponding layer material defines the LIDT of the layer system, and the layer most vulnerable to damage is responsible for the damage resistance of the entire layer system. Further on, it is well known that an increased silica content in the high refractive index material leads to an improved power handling capability [3]. Thereby, the change of the material composition within the refractive index step down (RISED) concept offers an additional option for improving the LIDT. The RISED concept is based on stabilization of the stack by substitution of high refractive index layers of lowest internal damage threshold by composite materials with a sufficient fraction of silica [3]. For binary stacks the internal LIDT scales with the internal field strength in the high refractive index layers. Technically, such ternary composites are manufactured applying well approved Ion Beam Co-sputtering.

However the production of CMs is very sensitive to marginal errors in layer thickness and/or in refractive index leading often to a total failure of the aimed phase target for the mirror and with respect to the technological state of the coating processes only the manufacturing of binary materials are available. Consequently, present monitoring strategies are

*t.willemsen@1zh.de; phone +49 (0) 511 27788-294; fax +49 (0) 511 27788-100; www.lzh.de

(C) 2016 SPIE $\cdot$ CCC code: $0277-786 X / 16 / \$ 18 \cdot$ doi: $10.1117 / 12.2244835$ 
challenged to their physical limits. Both, the accuracy of broad band and single wave length monitors are typically not high enough to manufacture CMs with the required phase conditions. Especially, the self-error-compensation of the BBM is not working with respect to the GD and the GDD. With respect to the sputtering of dielectric ternary composites, small positioning errors of a few $\mathrm{mm}$ of the zone target cause a change of the index of refraction.

Taking advantages of a novel in-situ monitoring concept it is possible to monitor the phase in reflectance directly during deposition as an extension of a conventional in-situ optical transmittance or reflection measurements applying a broad band monitor [BBM]. The current system is based on a Michelson interferometer in the NIR-wavelength range [13]. In previous studies it was shown that the monitored phase in the NIR range can be used to improve significantly the fabrication of CM`s in the NIR as well as in other spectral wavelength ranges as for instance in the UV [4; 13].

A new method is presented to fabricate dielectric dispersion compensating mirrors applying ternary composites inside the layer stack. The study is initiated by a design synthesis, to achieve optimum reflection and GDD values. Afterwards the two most suitable designs are manufactured applying an Ion Beam Sputtering (IBS) process. Both have similar target specifications with respect to the spectral and phase properties, whereby one design is using ternary composites and the other one is distinguished by a conventional high low stack. The first layers of the stack are switched applying in-situ broad band monitoring in conjunction with a forward re-optimization algorithm, which also manipulates the layers remaining for deposition at each switching event [5]. To accomplish the demanded GDD-spectra, the last layers are controlled by a novel in-situ white light interferometer operating in the infrared spectral range. Finally the CMs are measured in the USP LIDT in a 10.000 on1 procedure according to ISO 21254 [6] applying pulse duration of $130 \mathrm{fs}$ at a central wavelength of $775 \mathrm{~nm}$.

\section{DESIGN CONSIDERATIONS}

The presented design studies were preformed applying OptiLayer software [7]. In order to reduce the sensitivity of the design to deposition errors during the manufacturing process and to retrieve an optimum field distribution, the robust synthesis procedure using needle optimization and gradual evolution algorithms was used for the design calculation [8]. The given design target specifications were set to a group delay dispersion (GDD) of $-50 \mathrm{fs}^{2}$ within a spectral range of $100 \mathrm{~nm}$ in between $(720-820) \mathrm{nm}$ and a reflection greater than $99.5 \%$ for p-polarized laser light under an angle of incidence of $45^{\circ}$, respectively. Basically in a first step the best suited design is calculated applying high and low refractive index layers of Tantala $\left(\mathrm{Ta}_{2} \mathrm{O}_{5}\right)$ and Silica $\left(\mathrm{SiO}_{2}\right)$. This results in a design with a total physical thickness of 7.2 $\mu \mathrm{m}$ consisting out of 59 layers. The distribution of the field intensity $|\mathrm{E}|^{2}$ is presented in figure $1 \mathrm{a}$ ). Thereby $|\mathrm{E}|^{2}$ values are normalized to an incident wave of $|\mathrm{E}|^{2}=100 \%$. Since these values are quadratic, $|\mathrm{E}|^{2}$ can reach $400 \%$ in the incident medium for the case of conventional QWOT high reflectors [7].

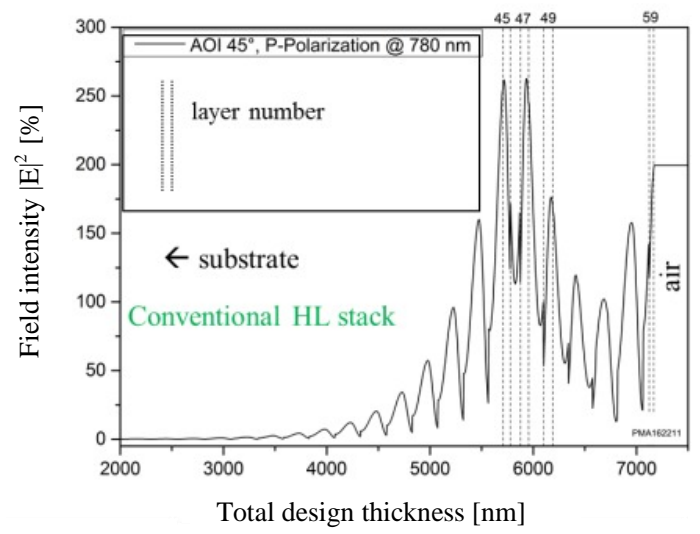

a) Design consisting of high and low index barriers. The horizontal dotted lines mark layers which will be substituted by ternary composites.

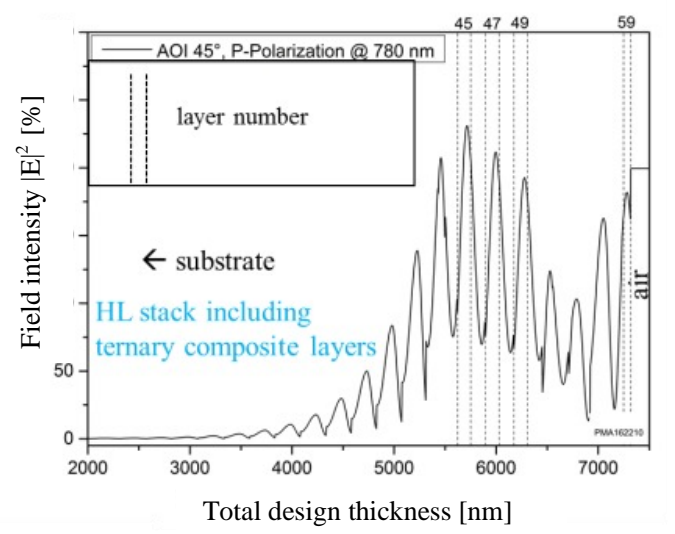

b) Refinement of the design presented in a) including ternary composite layers. The field intensity of the close by layer before the first composite barrier rises.

Figure 1. Field intensity of calculated dispersion compensating mirror designs. 
Highest intensities occur in Tantala layers deep inside the stack with a maximum of more than $260 \%$. These values define the laser induced damage threshold (LIDT) of the dielectric dispersive compensating mirror (CM). Four critical layers were identified and substituted by a fixed selected ternary composite of $\mathrm{Ta}_{\mathrm{x}} \mathrm{Si}_{\mathrm{y}} \mathrm{O}_{\mathrm{z}}$ characterized by an optical gap value of $4.75 \mathrm{eV}$ and a refractive index of 1.62 , respectively. This composition is expected to increase the LIDT while not leading to a strong decrease in the total reflectance of the layer stack.

Afterwards a refinement algorithm has to be applied to meet the GDD target. First, the total higher content of silica in the layer stack decreases the average reflectance by $0.1 \%$. Further, as a consequence of this refinement the field intensity $|\mathrm{E}|^{2}$ of other $\mathrm{Ta}_{2} \mathrm{O}_{5}$ layers in the stack rises, and defines the limit for the LIDT. The difference in $|\mathrm{E}|^{2}$ between this and the ternary composite layer with the highest field intensity $|\mathrm{E}|^{2}$ is about $30 \%$, which is expected to lead to an improvement in LIDT $[3,9,10]$. The detailed specifications of both mirrors are listed in table 1.

Table 1. Comparision of the two calculated chirped mirror designs

\begin{tabular}{|c|c|c|}
\hline & Conventional HL stack & HL stack including ternary composites \\
\hline Number of layers & 59 & 59 \\
\hline Total design thickness $[\mu \mathrm{m}]$ & 7.2 & 7.3 \\
\hline Spectral range $\Delta \lambda[\mathrm{nm}]$ & $720-820$ & $720-820$ \\
\hline Reflectance within $\Delta \lambda[\%]$ & $>99.7$ & $>99.6$ \\
\hline GDD within $\Delta \lambda\left[\mathrm{fs}^{2}\right]$ & -50 & -50 \\
\hline Polarization and AOI $\left[{ }^{\circ}\right]$ & p-pol under 45 & p-pol under 45 \\
\hline $\begin{array}{c}\text { Maximum field intensity of } \\
\mathrm{Ta}_{2} \mathrm{O}_{5}[\%]\end{array}$ & 260 & 230 \\
\hline
\end{tabular}

\section{DEPOSITION PROCESS AND LIDT MEASUREMENT PROCEDURE}

The mirrors were fabricated employing an ion beam co-sputtering process. The ion beam (Veeco $6 \mathrm{~cm}$ source, current $150 \mathrm{~mA}$, acceleration voltage $800 \mathrm{~V}$ ) is extracted from an Argon gas plasma by a three gird system and is directed on a zone target assembly. The target stage carries two targets of the materials Tantalum and Silicon, and it can be moved perpendicularly to the ion beam. Depending on the target position various overlapping fractions of the metals can be sputtered. The layers are deposited in a reactive oxygen atmosphere of $4 \times 10^{-4}$ mbar to achieve a well-balanced stoichiometry. The layer stacks are deposited on $1 \mathrm{~mm}$ thick fused silica substrates (1inch diameter) of standard quality. The thickness of each layer is controlled online by a broad band optical monitoring system (BBM) [11]. Applying a conventional halogen lamp, installed below the substrate mount, in combination with a fiber optic CCD spectrometer (Avantes), a wavelength range from $420 \mathrm{~nm}$ up to $1050 \mathrm{~nm}$ is covered. A schematical drawing of the setup is presented in fig. 2. 


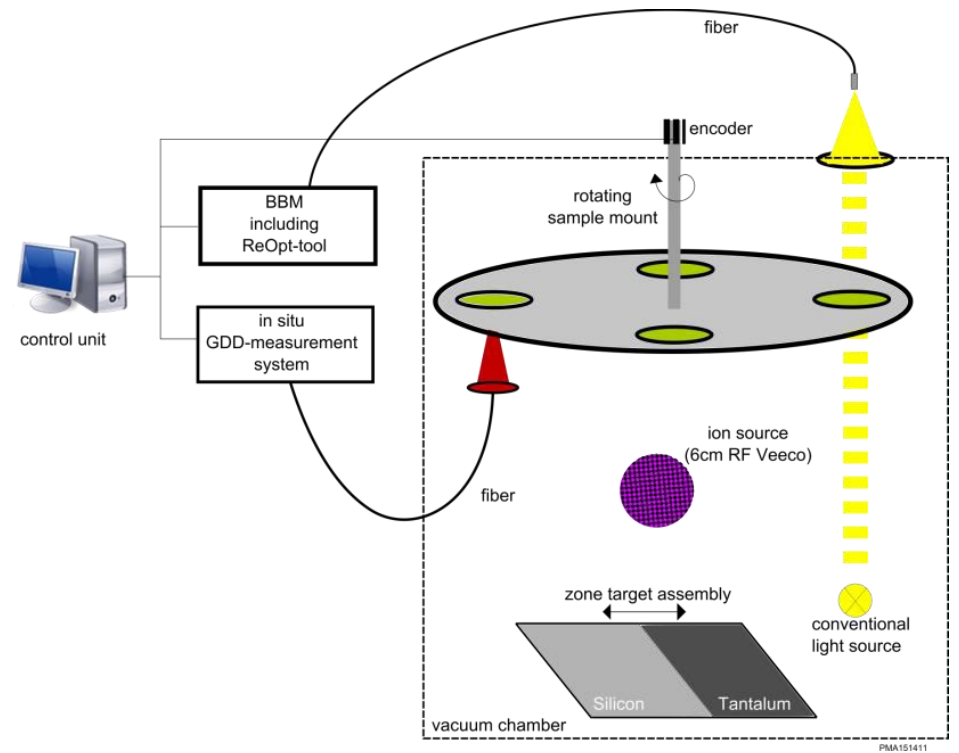

Figure 2. Schematic drawing of the deposition plant. A conventional IBS Co sputter process is applied for manufacturing $\mathrm{Ta}_{2} \mathrm{O}_{5}-\mathrm{SiO}_{2}$ layer stacks, which are controlled by a BBM using transmittance spectra. Deposition errors are corrected online by a re-optimization tool (ReOpt) applying the transmission data recorded after termination of each layer. The last layers of the dispersion compensating mirrors are terminated by the in situ group delay dispersion (GDD) measurement system, which mainly consists of a fiber based white light interferometer (WLI) and a fast spectrometer [13].

Possible discrepancies in particular layers can be corrected online during the coating process applying an in-house developed re-optimization tool [12]. Employing a needle algorithm, the subsequent layers can be optimized with respect to the specified group delay dispersion and reflection target values. An optimization cycle can be performed in situ after every coated layer [12]. Especially taking into account the high sensitivity of the designed GDD to the film thickness accuracy in the final layers, a novel monitoring strategy is applied. In this algorithm, the conventional BBM is used for layer termination until the fifth last layer of the design is finished, and afterwards the aligned interference of the novel in situ white light interferometer (WLI) is used to control and switch the last layers [13]. Starting the coating process again the last layers are terminated by the described in situ GDD measurement system. In summary the fiber based WLI in combination with a fast high resolution spectrometer displays the current in situ group delay dispersion curve every time the moving sample is passing the collimator head. The detailed setup and principle is presented in [13]. For a precise layer termination the current in situ GDD measurement curve is continuously compared to the theoretic design GDD curve of the particular layer. The available wavelength range of the in-situ phase monitor covers $810 \mathrm{~nm}$ up to $870 \mathrm{~nm}$ under an angle of incidence (AOI) of $0^{\circ}$, which does not cover the requirements of the application (AOI $45^{\circ}$ at $725 \leq \lambda \leq 825 \mathrm{~nm}$ ). But as mentioned, the algorithm allows to extrapolate the spectral and GGD data.

Furthermore, the LIDT was determined according to ISO 21254. Thereby the samples are irradiated by p-polarized laser pulses characterized by a central wavelength of $\lambda_{\mathrm{z}}=775 \mathrm{~nm}$, a pulse duration of $\tau_{\mathrm{P}}=130 \mathrm{fs}$ and an effective beam diameter $d_{\text {beam }}=97 \mu \mathrm{m}$. The LIDT value at the threshold of $0 \%$ was depicted after the sample was irradiated by 20000 pulses.

\section{RESULTS}

As it is presented in fig. 3 a) and b), the final measurement data (green solid line) applying white-light interferometry were compared to the theoretical design curve calculated for an AOI of $0^{\circ}$ (grey solid line). Based on the transmission data and retrieved GDD data from the in-situ phase monitor after every layer a reversed engineering was performed for $0^{\circ}$ (grey dotted line). Regarding the deviation of the measured GDD spectra (green solid line in fig 3a) and b)) in comparison to the calculated reverse engineering GDD spectra (grey dotted line in fig 3a) and b)), respectively, an approximately identical residual sum of squares $\operatorname{RSS}=\sum_{i=1}^{n}\left(G D D_{W L I}-G D D_{R e v}\right)^{2}$ is resulting. Further taking into 
account an $\mathrm{AOI}$ of $45^{\circ}$, the retrieved reverse engineering (grey dotted line) data were compared to the spectra of the original design for p-polarized light (black solid line in fig. $3 \mathrm{c}$ ) and d)). For both, the CM applying conventional (HL) ${ }^{\mathrm{n}}$ stacks (fig.3 c)) and the CM including ternary composites (fig. 3d)) a GDD of $(-50 \pm 30) \mathrm{fs}^{2}$ is reached (AOI $\left.45^{\circ}\right)$.

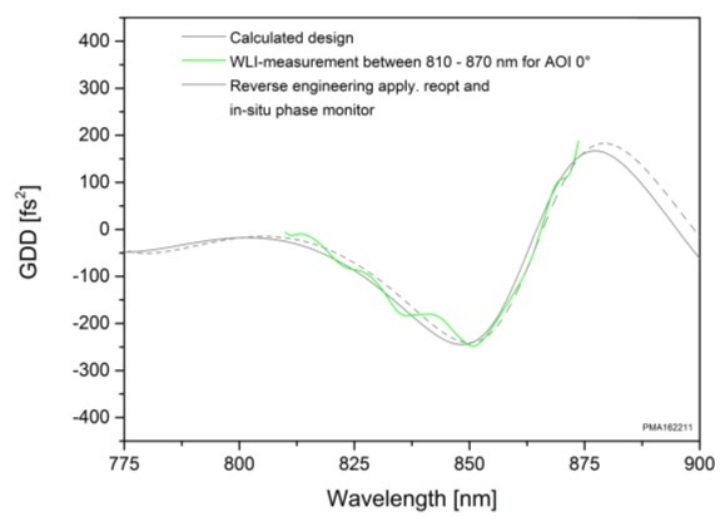

a) Conventional $(\mathrm{HL})^{\mathrm{n}}$ stack: Calculated designs compared to measurement for an AOI $0^{\circ}$

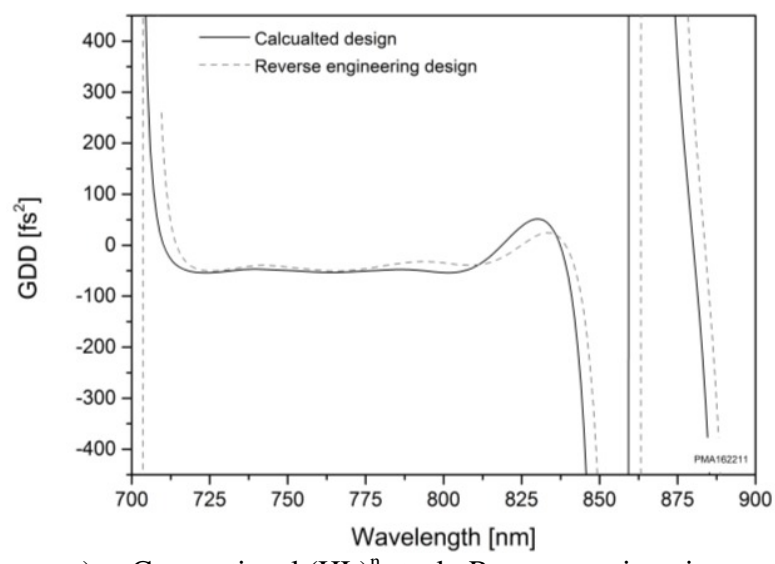

c) Conventional $(\mathrm{HL})^{\mathrm{n}}$ stack: Reverse engineering data retrieved from BBM and in situ phase monitor compared to calculated design (AOI45 $5^{\circ}$ p-polarized)

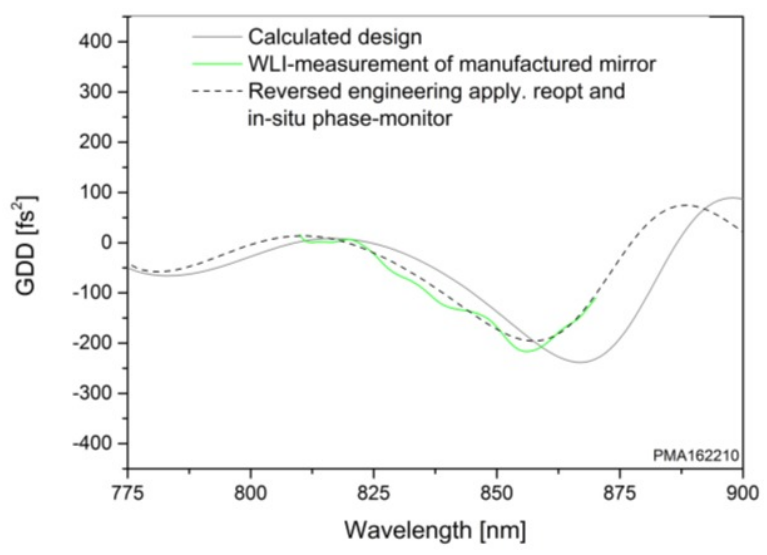

b) HL stack including ternary composite layers: Calculated designs compared to measurement for an AOI $0^{\circ}$

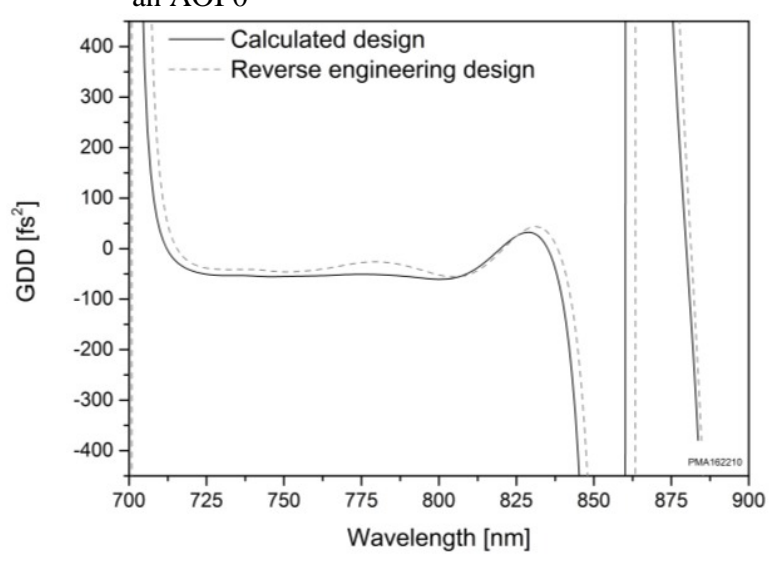

d) HL stack including ternary composite layers: Reverse engineering data retrieved from BBM and in situ phase monitor compared to calculated design ( $\mathrm{AOI} 45^{\circ}, \mathrm{p}$-polarized)

Figure 3. Group delay dispersion measurements are compared to the reverse engineering design data.

In a next step the LIDT of both samples were determined and compared (fig. 4). The corresponding LIDT values are set at 20000 pulses to reduce measurement uncertainties which are bigger for lower pulse classes (fig. 4 a) and b)). An LIDT of $0.23 \mathrm{~J} / \mathrm{cm}^{2}$ (0\% threshold @20000 pulses) results for the conventional CM (fig. 4c). However, analysing the determined LIDT of the CM applying ternary composite layers an LIDT improved by more than $40 \%$ can be presented (fig. $4 \mathrm{~d}$ ), which is corresponding to an absolute value of $0.32 \mathrm{~J} / \mathrm{cm}^{2}$. 


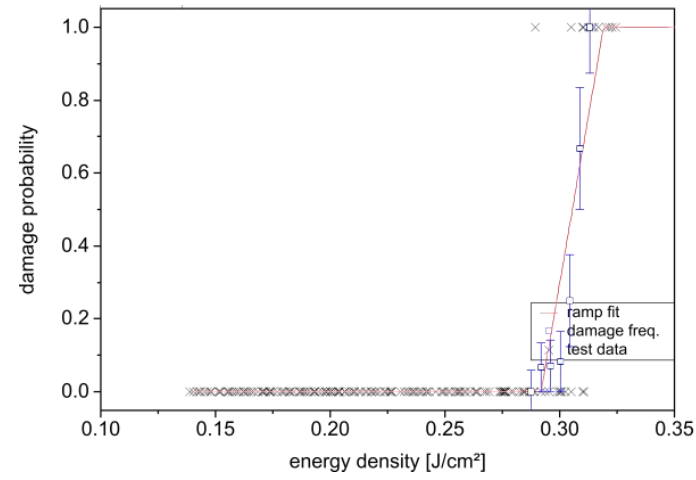

a) Damage probability after irradiation of 10 pulses for the conventional $(\mathrm{HL})^{\mathrm{n}}$ chirped mirror



c) Chirped mirror applying conventional $(\mathrm{HL})^{\mathrm{n}}$ stack

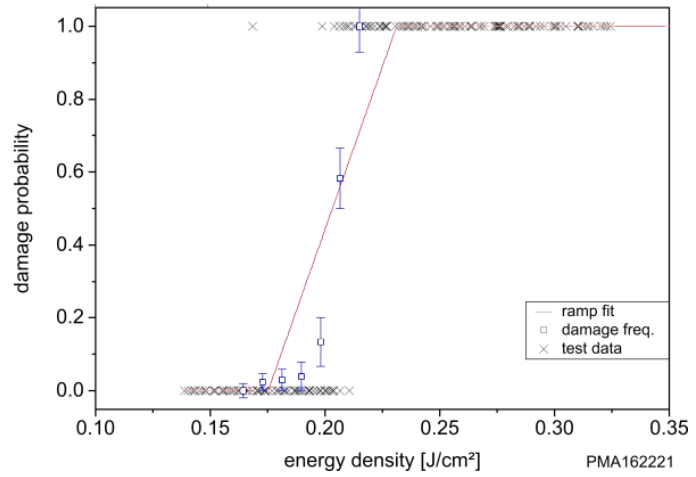

b) Damage probability after irradiation of 20000 pulses for the conventional $(\mathrm{HL})^{\mathrm{n}}$ chirped mirror



d) Chirp mirror applying ternary composite layers

Figure 4. LIDT measurements of the two tested chirped mirrors. The mirror applying ternary composite layers is characterized by a $40 \%$ higher LIDT value.

\section{CONCLUSION}

In this study a new approach is presented to improve the laser induced damage threshold (LIDT) of dispersive compensating mirrors (CMs) applying ternary composite layers of $\mathrm{Ta}_{\mathrm{x}} \mathrm{Si}_{\mathrm{y}} \mathrm{O}_{\mathrm{z}}$. The contribution is initiated by an analysis of the complex field intensity $|\mathrm{E}|^{2}$ distribution of a calculated CM design of a conventional (HL) ${ }^{\mathrm{n}}$ stack. This design is characterized by the specifications of a spectral range in between $(720-820) \mathrm{nm}$ under an AOI of $45^{\circ}$. Four critical $\mathrm{Ta}_{2} \mathrm{O}_{5}$ layers inside the stack were identified to limit the LIDT because of highest field intensity. These layers are substituted by fixed ternary composite layers of $\mathrm{Ta}_{\mathrm{x}} \mathrm{Si}_{\mathrm{y}} \mathrm{O}_{\mathrm{z}}\left(\mathrm{E}_{\mathrm{gap}}=4.75 \mathrm{eV} \mathrm{n}_{800 \mathrm{~nm}}=1.62\right)$ according to an initial step of RISED concept. A necessary refinement of the design to smoothen the group delay dispersion target increased the field intensity of close by $\mathrm{Ta}_{2} \mathrm{O}_{5}$ of the first composite layer. This resulting difference of $\Delta|\mathrm{E}| \sim 30 \%$ is expected to lead to an improvement in LIDT for the comparable CM applying ternary composite layers $[9,10]$. However the deposition remains very sensitive to minute deposition errors. Taking advantage of a novel in-situ optical monitor, the phase in reflectance could be monitored directly during deposition, and the last layers of both designs were switched applying this technique. Ex-situ white light interferometry measurements of both fabricated mirrors displayed a very high agreement to the calculated design, and reverse engineering data confirmed the desired target specifications. Afterwards the samples were irradiated by laser pulses $\left(\tau_{\mathrm{P}}=130 \mathrm{fs} ; \lambda_{Z}=775 \mathrm{~nm}\right.$; AOI $45^{\circ}$; p-polarized) to determine the LIDT. An experimental improved LIDT of $40 \%$ could be shown for the similar CM applying ternary composite layers. In future studies the RISED concept can be applied for a further increasing of the LIDT. Thereby improvements up to the quadruple can be expected comparable to optimizations of HR mirrors [3]. 


\section{ACKNOWLEDGEMENT}

This work was supported by the Deutsche Forschungsgemeinschaft (DFG) within the cluster of excellence 201 Quest and the Volkswagen Stiftung of the research project "Hymnos" (contract no. ZN3061). The authors also thank the Federal Ministry of Education and Research (BMBF) for the financial support within the research project Ultra-LIFE under grant $13 \mathrm{~N} 11558$.

\section{REFERENCES}

[1] Szipöcs, R., Spielmann, C., Krausz, F., and Ferencz, K., "Chirped multilayer coatings for broadband dispersion control in femtosecond lasers," Optics Letters, 19(3), 201-203 (1994).

[2] Pervak, V., Razskazovskaya, O., Angelov, I. B., Vodopyanov, K. L., and Trubetskov, M., "Dispersive mirror technology for ultrafast lasers in the range 220-4500 nm," Advanced Optical Technologies, 3(1), 55-63 (2014).

[3] Ristau, D. (Ed.). (2014). Laser-Induced Damage in Optical Materials. CRC Press.

[4] Willemsen, T., Schlichting, S., Kellermann, T., Jupé, M., Ehlers, H., Morgner U., and Ristau D., "Precise fabrication of ultra violet dielectric dispersion compensating mirrors," Proc. of SPIE 9627, 96271U (2015).

[5] Amotchkina, T. V., Trubetskov, M. K., Pervak, V., Schlichting, S., Ehlers, H., Ristau, D., and Tikhonravov, A. V., "Comparison of algorithms used for optical characterization of multilayer optical coatings," Applied Optics, 50(20), 3389-3395 (2011).

[6] ISO 21254, "Lasers and laser-related equipment - Test methods for laser-induced damage threshold," (2011).

[7] Tikhonravov, A.V., and Trubetskov, M.K., "OptiLayer software," http://www.optilayer.com

[8] Pervak, V., Trubetskov, M. K., and Tikhonravov, A. V., "Robust synthesis of dispersive mirrors," Optics Express, 19(3), 2371-2380 (2011).

[9] Starke, K., Gross, T., Ristau, D. "Laser-induced damage investigation in chirped mirrors for ultrashort-pulse laser systems". Proc. SPIE 4347, 528 (2000)

[10] Neauport, J., Lavastre, E., Razé, G., Dupuy G., Bonod, N., Balas, M., de Villele, G., Flamand, J., Kaladgew, S., and F. Desserouer, "Effect of electric field on laser induced damage threshold of multilayer dielectric gratings," Opt. Express 15, 12508-12522 (2007)

[11]Ristau, D., Ehlers, H., Gross, T., and Lappschies, M., "Optical broadband monitoring of conventional and ion processes," Applied Optics, 45(7), 1495-1501 (2006).

[12] Schlichting, S., Heinrich, K., Ehlers. H., Ristau, D., "Online re-optimization as a powerful part of enhanced strategies in optical broadband monitoring," Proc. of SPIE 8168, 81681E (2011).

[13] Schlichting, S., Willemsen, T., Ehlers, H., Morgner, U. and Ristau, D., "Fourier-transform spectral interferometry for in situ group delay dispersion monitoring of thin film coating processes," Opt. Express 24, 22516-22527 (2016) 